\title{
Shifts in morphology, gene expression, and selection underlie web loss in Hawaiian Tetragnatha spiders
}

\author{
Cory A. Berger ${ }^{1,2^{*}}$ (D) Michael S. Brewer ${ }^{3}$, Nobuaki Kono ${ }^{4}$, Hiroyuki Nakamura ${ }^{5}$, Kazuharu Arakawa ${ }^{4}$, \\ Susan R. Kennedy ${ }^{6}$, Hannah M. Wood ${ }^{7}$, Seira A. Adams ${ }^{1 \dagger}$ and Rosemary G. Gillespie ${ }^{1+}$
}

\begin{abstract}
Background: A striking aspect of evolution is that it often converges on similar trajectories. Evolutionary convergence can occur in deep time or over short time scales, and is associated with the imposition of similar selective pressures. Repeated convergent events provide a framework to infer the genetic basis of adaptive traits. The current study examines the genetic basis of secondary web loss within web-building spiders (Araneoidea). Specifically, we use a lineage of spiders in the genus Tetragnatha (Tetragnathidae) that has diverged into two clades associated with the relatively recent ( 5 mya) colonization of, and subsequent adaptive radiation within, the Hawaiian Islands. One clade has adopted a cursorial lifestyle, and the other has retained the ancestral behavior of capturing prey with sticky orb webs. We explore how these behavioral phenotypes are reflected in the morphology of the spinning apparatus and internal silk glands, and the expression of silk genes. Several sister families to the Tetragnathidae have undergone similar web loss, so we also ask whether convergent patterns of selection can be detected in these lineages.
\end{abstract}

Results: The cursorial clade has lost spigots associated with the sticky spiral of the orb web. This appears to have been accompanied by loss of silk glands themselves. We generated phylogenies of silk proteins (spidroins), which showed that the transcriptomes of cursorial Tetragnatha contain all major spidroins except for flagelliform. We also found an uncharacterized spidroin that has higher expression in cursorial species. We found evidence for convergent selection acting on this spidroin, as well as genes involved in protein metabolism, in the cursorial Tetragnatha and divergent cursorial lineages in the families Malkaridae and Mimetidae.

Conclusions: Our results provide strong evidence that independent web loss events and the associated adoption of a cursorial lifestyle are based on similar genetic mechanisms. Many genes we identified as having evolved convergently are associated with protein synthesis, degradation, and processing, which are processes that play important roles in silk production. This study demonstrates, in the case of independent evolution of web loss, that similar selective pressures act on many of the same genes to produce the same phenotypes and behaviors.

Keywords: Convergence, Tetragnatha, Spidroins, Web loss, Transcriptomics, Selection, Gene expression, Hawai ${ }^{\dagger} \mathrm{i}$

\section{*Correspondence: caberger@mit.edu}

${ }^{\dagger}$ Seira A. Adams and Rosemary G. Gillespie shared senior authors

${ }^{1}$ Department of Environmental Science, Policy and Management, University of California, Berkeley, 130 Mulford Hall, \#3114, Berkeley, CA 94720-3114, USA

Full list of author information is available at the end of the article

\section{Background}

Convergent evolution occurs across many scales of biological complexity, from molecules to behavior, and across phylogenetic scales, from species to genera and families [1]. Some of the best examples of convergence are found in archipelago settings, where similar sets of ecological forms have evolved mostly independently on 
the constituent islands, e.g. Anolis lizards in the Caribbean [2] and Hawaiian spiders [3]. Convergence also produces similar ecological forms at much deeper phylogenetic scales, with well known examples in Australian marsupials that have convergent counterparts among placental mammals in other parts of the world [4]. In both these situations, geographic isolation leads to the convergence of sets of species that look similar when comparing across different geographical settings (e.g. Australian thylacines and American canids), but are most closely related to species that are divergent in ecology and live in the same area. In other situations, convergence is imposed by similar selective pressures across transitions to marine [5] or high altitude [6] environments, or with the adoption of phenotypes such as social behavior [7] and echolocation [8]. In these cases, convergence is associated with repeated gains of a suite of traits. In other environments convergence can lead to trait regression, or the tendency of organisms to lose traits when released from selection for those traits, particularly when the traits are costly. For example, birds and insects frequently become flightless on islands [9], and cave organisms tend to lose eyes and pigmentation [10]. Loss of structures may be associated with neutral evolution-the accumulation of random mutations in genes associated with the regressed trait-or natural selection, which can be positive (e.g. energy gain as a result of loss of the trait [11]) or negative (the trait is deleterious). Therefore, a critical step in understanding the evolution of convergent traits, regressive or otherwise, is not only linking behavior to morphology, but linking both to the molecular basis for a given trait [12]. In particular, in cases where similar phenotypes are displayed by distantly related taxa, to what extent can alternative genetic pathways operate to create similar suites of traits [13], and at what point in the formation of the phenotype [1]?

The current study examines the phenomenon of web loss in a lineage of orb-web spiders in order to understand the relationship between behavior, morphology, and the genomic basis of the web-spinning trait. Spiders provide an ideal system for linking functional morphology (spinneret spigots and silk glands) and behavior (web building) to specific changes in the genome in order to understand how selection operates on complex traits (e.g. $[14,15])$, building upon existing knowledge of the genetic basis of silk production. Spider silk fibers are primarily made up of proteins called spidroins-a contraction of "spider fibroin" [16]. Spidroins are typically large proteins with a unique structure consisting of long regions of tandem repeats flanked by non-repetitive amino and carboxyl (N- and C-) terminal domains [16, 17]. Studies have shown a clear correlation between expression at the transcriptome level and the demonstration of web spinning behaviors [18]. Moreover, adoption of a cursorial lifestyle can lead to changes in silk expression, such as increased production of major ampullate (dragline) silk [19].

The evolution of spider silk has received considerable attention [17, 20-23]. More derived spiders possess multiple distinct silk glands, which are serially homologous. These appear to have diversified through duplication, attaining different properties over their evolutionary histories [23]. Each silk gland comprises a sac, which connects through a duct of variable length and diameter to an excretory duct or spigot, located on a spinneret [24]. Within the sac of each gland, the spider synthesizes a fiber or glue with specific mechanical properties that are the result of gland-specific gene expression [16]. Within the superfamily Araneoidea, adult females can have up to seven types of silk glands-piriform (attachment cement), aciniform (wrapping), tubuliform (egg sac covering), major ampullate (dragline), minor ampullate (temporary spiral), aggregate (sticky glue) and flagelliform (capture spiral); the silk associated with each type of gland is extruded through a corresponding spigot [24, 25]. The spigots associated with spinning these different silks have well-defined morphologies and placement within the spinning fields on the spider spinnerets. Thus, the anterior lateral spinnerets (ALS) contain the major ampullate spigot and numerous piriform spigots; the posterior medial spinnerets (PMS) contain the minor ampullate spigot, a tubuliform spigot, and numerous aciniform spigots; and the posterior lateral spinnerets (PLS) contain another tubuliform spigot, numerous aciniform spigots, two aggregate spigots, and a single flagelliform spigot $[25,26]$. The spinning of sticky capture silk is associated with a morphological structure located on each PLS: the "aggregate/flagelliform triad" consisting of two aggregate spigots and one flagelliform spigot [26]. This triad is a key synapomorphy of spiders in the superfamily Araneoidea $[26,27]$. The flagelliform gland, and associated single spigot, produces a core line while the silk from the aggregate gland spigots coats this line with glue; the ducts of the aggregate and flagelliform glands are also physically attached [28]. In structure, the flagelliform glands consist of a long duct with a cylindrical proximal portion, similar to the ampullate glands, and are variable in size, smaller in the Linyphiidae and Theridiidae relative to the Araneidae, each of these families being closely related to Tetragnathidae [24]. The two pairs of aggregate glands are unusual-large, multilobed structures that appear to have been derived from coalescence of a number of smaller glands [24].

Although the sticky capture threads, and associated well-developed aggregate and flagelliform triad, are characteristic of the Araneoidea, the use of the sticky capture threads is frequently lost, and with it the aggregate and 
flagelliform triad. In most araneoids, adult males adopt a vagrant lifestyle and the triplet is lost [28] or vestigial $[29,30]$ in the final instar. Moreover, there are multiple independent cases where entire lineages have abandoned web building, and these cases are also accompanied by loss of the aggregate/flagelliform triad [26, 31-35]. Thus, it appears that convergent trait regression, with repeated loss of the sticky spiral triad of spinneret spigots, is tied to the adoption of a cursorial lifestyle. However, it is not clear whether these convergent phenotypic changes are reflected at the molecular level, and hence the relationship between genotype and phenotype remains unknown. In particular, given that the trait is convergent, are the morphological and behavioral similarities the result of similar changes at the molecular level, and are the selective pressures also acting in a similar way?

The current study examines convergent trait regression associated with the adoption of a cursorial lifestyle within the family Tetragnathidae, and asks whether changes in selection act on the same genes as in the related families of Mimetidae, Malkaridae, and Arkyidae, which are characterized by a similar adoption of a cursorial lifestyle and the absence of the aggregate/flagelliform triad [33]. The family Tetragnathidae-and presumably the ancestors of Mimetidae, Malkaridae, and Arkyidae-share many behavioral and morphological synapomorphies with other members of Araneoidea, including the presence of the aggregate/flagelliform triad for producing viscid sticky silk, and the movements involved in orb web construction [26]. However, partial loss of the triad has been documented in multiple lineages within the Tetragnathidae. The current study focuses on another tetragnathid lineage, a large species radiation in Hawai' $i$ within the genus Tetragnatha [36]. This lineage appears to be monophyletic, with early divergence, ca. 5 million years ago [36], into two major clades. One of these clades has retained the web-spinning behavior that characterizes the genus, while the other, known as the "Spiny Leg" clade, has abandoned web building and adopted a cursorial lifestyle [37]. The parallel radiation of these two lineages provides an ideal opportunity to compare selective pressures acting on each clade. We examine how changes in the behavior of the Spiny Leg lineage relative to web spinners are reflected in (1) the morphology of the silk spinning apparatus, in particular the aggregate/flagelliform triad; (2) the presence of the silk glands themselves; and (3) changes in gene expression. Finally, we ask (4) whether natural selection has acted convergently in Spiny Leg Tetragnatha and in the clades Mimetidae, Malkaridae, and Arkyidae, where web loss has occurred independently. We sequenced six new transcriptomes and conducted a transcriptomic analysis between web building and non-web building members of the genus
Tetragnatha, then tested for evidence of selection associated with web loss and the associated adoption of a cursorial lifestyle across the Tetragnatha phylogeny. We also tested for convergent selection using available transcriptomes from Mimetidae, Malkaridae, and Arkyidae.

\section{Results}

\section{Morphology of spinneret spigots and silk glands}

The most remarkable feature of the posterior lateral spinnerets is the very obvious presence of the aggregate and flagelliform spigots in all seven species of web-spinners (Fig. 1); these spigots form a triad associated with the production of sticky silk used in web-building. However, the four species in the Spiny Leg clade all demonstrate a complete absence of the aggregate and flagelliform spigots. Dissections were perfomed on three web-building (T. stelarobusta, T. filiciphilia, T. paludicola) and three Spiny Leg species (T. brevignatha, T. quasimodo, T. waikamoi); aggregate glands were easily identified as large, multilobed, semi-transparent white structures in all webbuilders, and were found in none of the three Spiny Leg species (Fig. 2). Flagelliform glands are much smaller and more difficult to identify [24]. For this reason, we are not able to say with confidence that flagelliform glands are definitively absent from Spiny Leg spiders; however, aggregate glands have clearly been lost.

\section{Transcriptome assemblies}

We have assembled the first protein-coding transcriptomes of six species of Hawaiian Tetragnatha spiders. We generated twelve RNA-seq libraries from the dissected abdomens of adult female spiders (two individuals per species); library and assembly statistics are given in Additional file 1. Sequencing depth ranged between 20.6 and 36.5 million paired-end 100 bp reads. Quality filtering removed an average of $6.57 \%$ of raw reads. After trimming, libraries contained between 19 and 33 million paired-end reads per individual, or between 43 and 62 million reads per species, with fragment lengths between 50 and $101 \mathrm{bp}$. De novo assemblies made with Trinity contained between 101,722 and 122,506 transcripts with a minimum contig length of $201 \mathrm{bp}$. The GC content of each species assembly was $\sim 37 \%$. All libraries had greater than $80 \%$ of reads map as proper pairs to their respective assembly using Bowtie2. The six assemblies recovered between 96.4 and $97.8 \%$ of the BUSCO odb09 set of 1066 core arthropod genes, which is at the high end for de novo transcriptome assemblies and indicates good coverage of the protein-coding transcriptomes. After predicting coding sequences using TransDecoder and removing redundant peptides (at $100 \%$ identity) with CD-HIT, the assemblies contained between 23,774 and 28,202 nonredundant peptides. 


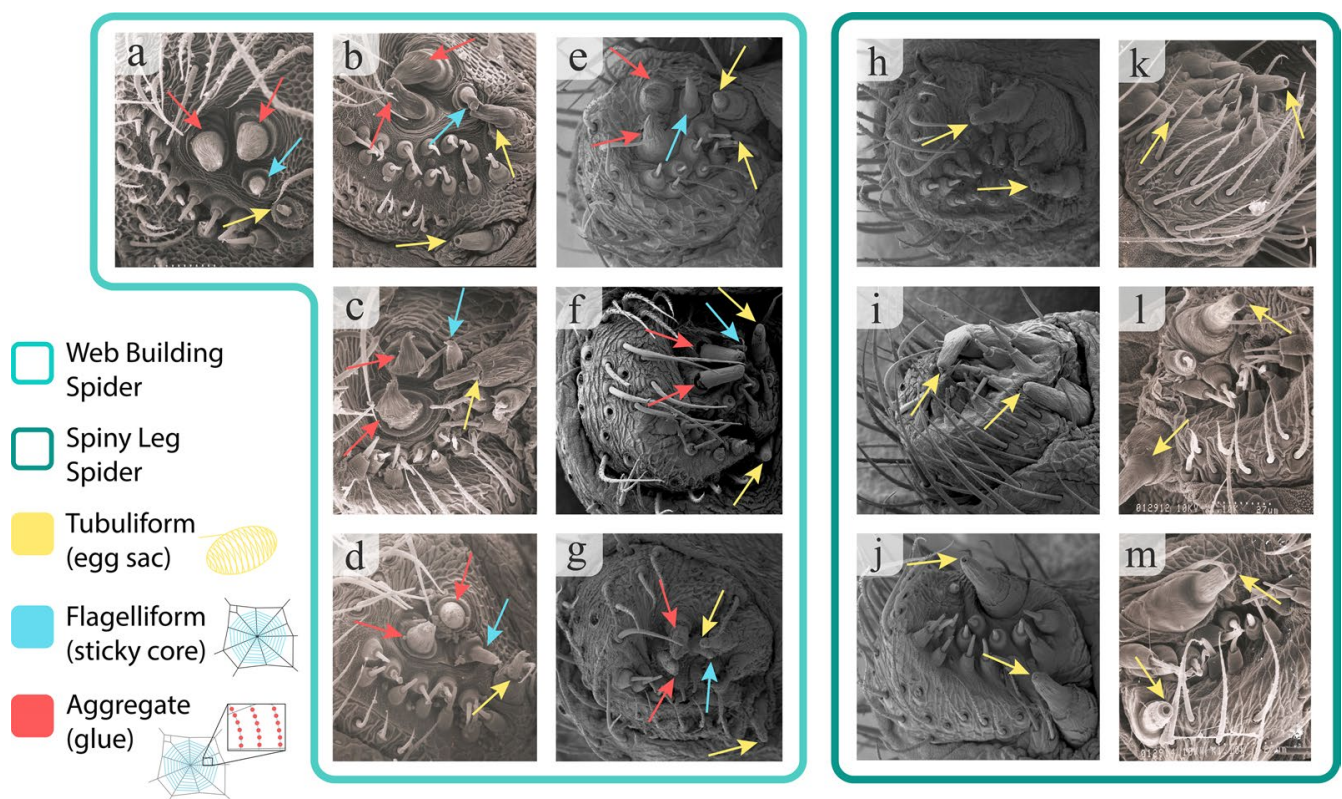

Fig. 1 Scanning Electron Microscopy Images of the posterior lateral spinnerets. Web builders (7) shown on the left: a Tetragnatha acuta, b T. trituberculata, c T. hawaiensis, d T. eurychasma (photos taken with protocol used in 1990), e T. sp. "Little wave", f T. stelarobusta, and $\mathbf{g}$ T. sp. "Long-clawed legs" (photos taken with protocol used in 2016). Spiny Leg clade (6) on the right: $\mathbf{h}$ T. kauaiensis, i T. quasimodo, j T. macracantha (photos taken with protocol used in 2016); and $\mathbf{k}$ and I T. waikamoi (2 individuals) and $\mathbf{m}$ T. quasimodo (photos taken with protocol used in 1990). Yellow arrows: tubuliform spigots; blue arrows: flagelliform spigots; green arrows: aggregate spigots. Unlabelled spigots are aciniform
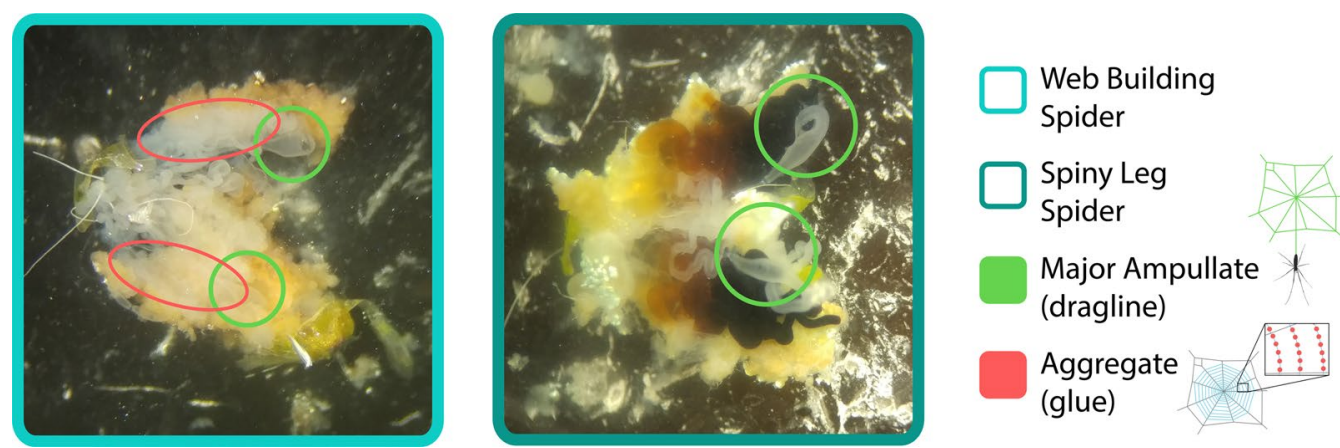

Fig. 2 Representative photographs of dissected abdomens showing silk glands. Aggregate glands (red) are large, multilobed, whitish structures that are only visible in web-building species, shown on the left. Major ampullate glands (green) are whitish but smaller, and have a distinctive duct shape that is easy to recognize. For simplicity, other glands are not labelled (or are too small to see). Yellowish lumps are adipose tissue. Left: Web builder, T. paludicola. Right: Spiny leg, T. brevignatha

\section{Spidroin phylogenetic analysis}

Full-length spidroins are difficult to assemble due to their unique structure, so we limited our analysis to $\mathrm{N}$ - and $\mathrm{C}$-terminal domain sequences. We searched the predicted peptides for spidroin terminal domains using BLAST and constructed Bayesian consensus trees for both sets of termini (Fig. 3). We identified sequences belonging to the seven major spidroin clades (aciniform, aggregate, flagelliform, major ampullate, minor ampullate, piriform, and tubuliform), as well as three uncharacterized spidroins with homology to genes that had previously been identified only in the orb-weaving genus Trichonephila (Sp5803, Sp-907/74867, and Sp-1339) [14, 38]. Phylogenetic conflict can occur between spidroin $\mathrm{N}$ - and C-termini, and $\mathrm{N}$-termini generally contain more phylogenetic signal [21, 39]. Nonetheless, the only major topological difference between our two trees is that the N-terminal tree recovers a sister relationship between aciniform and tubuliform (as in [21]), whereas the C-terminal tree places aciniform sister to (flagelliform + aggregate). Note 


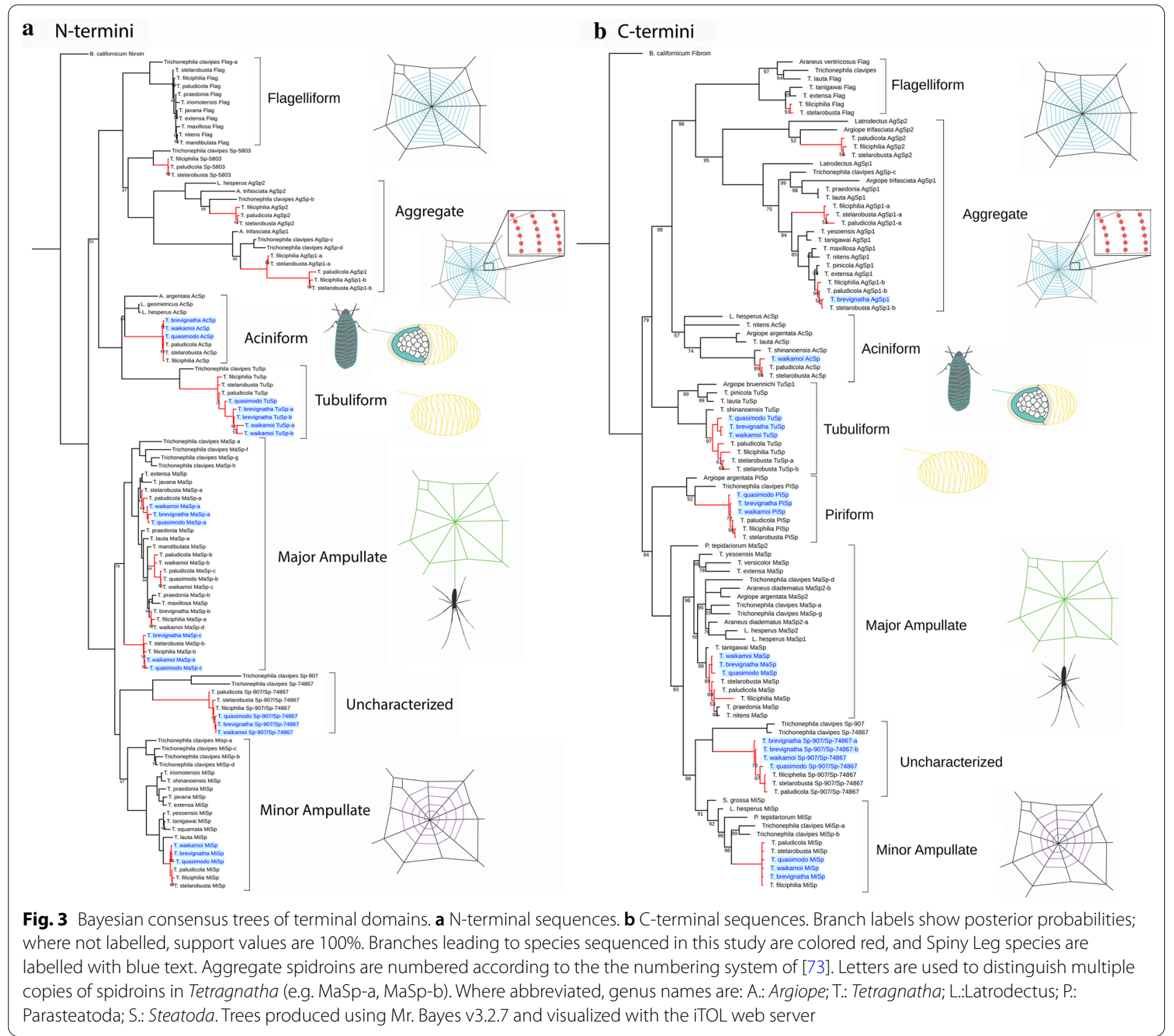

that with our data, we have no way of knowing which Nand $\mathrm{C}$-terminal sequences belong to the same physical gene. None of the three Spiny Leg transcriptomes contained sequences belonging to the flagelliform or Sp-5803 clades. Hawaiian Tetragnatha appear to possess three aggregate spidroin genes, two of which are expressed in some Spiny Leg species (Additional file 2).

\section{Differential expression analysis and gene ontology enrichment}

OrthoFinder assigned predicted proteins to 19,675 orthogroups, and PhyloTreePruner identified 7835 single-copy orthologs with genes in all six taxa, which were used for differential expression analysis between web builders and Spiny Legs. We identified 107 (1.4\%) differentially expressed (DE) single-copy orthologs; of these, 49 were more highly expressed in Spiny Legs and 58 were more highly expressed in web builders. $\mathrm{DE}$ genes and their annotations are given in Additional file 3. Seventeen out of the $107 \mathrm{DE}$ genes could not be annotated because they had no significant BLASTP hit against NCBI's nr database (accessed November $23,2020)$, or because the only hits were to hypothetical or uncharacterized proteins. One spidroin, Sp-907/ Sp-74867, was more highly expressed in Spiny Legs. Many of the remaining DE genes (both up- and downregulated) belong to groups of genes previously identified as having silk gland-specific expression (in [18, $38,40]$ ), including proteases, transferases, and genes associated with cellular transport and secretion; the 
expression of some of these genes is shown in Fig. 4 . Genes with higher expression in Spiny Legs include protease inhibitors such as zonadhesins, as well as transferases and transporter proteins. We observed reduced expression in Spiny Legs of some genes with aggregate gland-specific expression in other spiders, including an $\mathrm{N}$-acetylgalactosaminyltransferase (ppGalNAc-T) [41] and a globin protein [40]. Other genes with lower expression in Spiny Legs include an aminotransferase, a semaphorin membrane protein, and a cathepsin endopeptidase. Genes with higher expression in Spiny Legs were enriched for two gene ontology (GO) categories (FDR $<0.1)$ : "chitin binding" and "transferring acyl groups other than amino-acyl groups". Genes with higher expression in web builders were not enriched for any GO categories.

\section{Selection analyses}

The first approach using the FUSTr [42] pipeline identified eight gene families corresponding to spidroins: of these, two contained sites showing evidence for pervasive positive selection, an aggregate spidroin and a major ampullate spidroin (Table 1). Neither family included sequences from Hawaiian Tetragnatha. Five of the remaining six spidroins included sequences from nonweb building taxa and were further analyzed for selection along branches leading to those taxa. Three genes showed evidence for relaxed selection: an aggregate spidroin exhibited relaxed selection in the Spiny Leg T. tantalus, spidroin Sp-1339 exhibited relaxed selection in all Hawaiian Tetragnatha (web builders and Spiny Legs), and spidroin Sp-907/Sp-74867 exhibited relaxed selection in the non-web builders T. waikamoi, T. brevignatha, all analyzed species of Malkaridae, and Australomimetus sp. Spidroin Sp-907/Sp-74867 also showed evidence for episodic positive (diversifying) selection in Malkaridae. Finally, another aggregate sequence showed evidence for episodic positive selection in the Spiny Leg T. polychromata. For the second analysis using the COATS pipeline, the best-fit model involved positive selection along just the Spiny Leg branch for ten genes (Table 2; species tree used for COATS analysis shown in Fig. 5). These included ribosomal proteins and genes involved in protein ubiquitination and vesicle transport. For 24 genes, the best model involved selection in all non-web building taxa; these genes included mitochondrial enzymes,

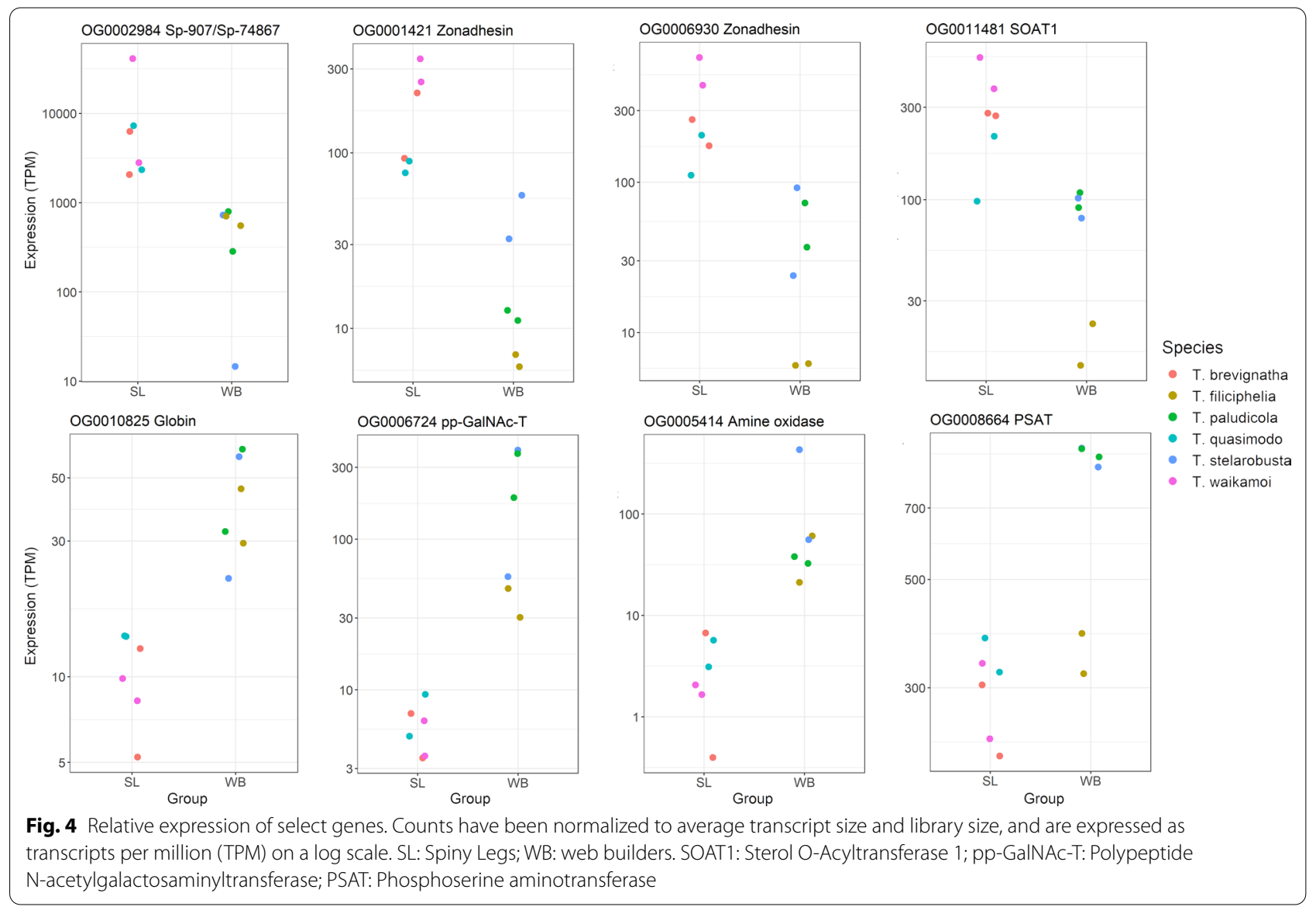


Table 1 Results of spidroin gene family selection analyses

\begin{tabular}{|c|c|c|c|c|c|}
\hline FUSTr family & Annotation & $\begin{array}{l}\text { Pervasive } \\
\text { selection } \\
\text { (FUBAR) }\end{array}$ & Episodic selection (aBSREL) & Relaxed selection (RELAX) & Relax K \\
\hline Family_5412 & Sp-907/Sp-74867 (C-terminal) & NA & Malkaridae sp. 17 & $\begin{array}{l}\text { Australomimetus sp., Malkaridae sp. } 17, T \text {. } \\
\text { brevignatha, T. waikamoi }\end{array}$ & $K=0.22$ \\
\hline Family_18703 & Sp-1339 (C-terminal) & NA & NA & $\begin{array}{l}\text { T. brevignatha, T. filiciphilia, T. paludicola, } T \text {. } \\
\text { perreirai, T. quasimodo, T. stelarobusta, } T \text {. } \\
\text { waikamoi }\end{array}$ & $K=0.31$ \\
\hline Family_19435 & AgSp (repetitive region) & NA & T. polychromata & NA & NA \\
\hline Family_21837 & AgSp (C-terminal) & NA & NA & T. tantalus & $K=0.09$ \\
\hline Family_68763 & MaSp (N-terminal) & 15 residues & NA & NA & NA \\
\hline Family_30334 & AgSp (C-terminal) & 3 residues & NA & NA & NA \\
\hline Family_54136 & AgSp (repetitive region) & NA & NA & NA & NA \\
\hline Family_9974 & AcSp (C-terminal) & NA & NA & NA & NA \\
\hline
\end{tabular}

For RELAX test, K parameter indicates degree of relaxation, with smaller values indicating greater relaxation in the test branches. AgSp, aggregate spidroin; MaSp, major ampullate spidroin; AcSp, Aciniform spidroin; Non-web building (cursorial) taxa are indicated in bold

ATPases, proteasome components, and additional genes related to protein synthesis and ubiquitination (Table 2). None of these 34 genes overlapped with the set of genes that were differentially expressed between Spiny Legs and web builders.

\section{Discussion}

The current study examined secondary loss of web building behavior within a radiation of Hawaiian spiders to determine the associations between behavior, morphology, and gene expression, specifically asking at which level selection appears to operate, and whether there is compensatory selection on other silks in association with the adoption of a cursorial lifestyle. We show that nonweb building "Spiny Leg" Tetragnatha have lost aggregate and flagelliform spigots, as well as at least the aggregate glands. These behavioral and morphological changes are accompanied by shifts in gene expression and branchspecific selection. Intriguingly, we identify an important role for a novel spidroin in web loss, as this gene shows higher expression and shifts in selection in non-web builders. We also detect evidence for convergent evolution between Spiny Leg Tetragnatha and other non-web building lineages, not only in morphology (loss of aggregate and flagelliform spigots), but at the genomic level, with selection acting on some of the same genes across independent web loss events.

\section{Characterizing the transition to cursoriality in Spiny Leg Tetragnatha Morphology of the silk spinning apparatus and associated silk glands}

The web building Hawaiian Tetragnatha examined in this study clearly show a well-developed triad of the aggregate and flagelliform spigots. However, these structures appear to be completely absent in the Spiny Leg taxa examined (Fig. 1). The absence of these spigots parallels similar losses that have been documented for the Mimetidae, Malkaridae, and Arkyidae s.l. [35] (although mimetids may retain flagelliform and aggregate spigots as juveniles [43]). The lack of the aggregate/flagelliform triad is accompanied by an apparent absence of the associated aggregate and flagelliform glands in the Spiny Leg taxa-while the large aggregate glands are clearly lost, it is possible that the small flagelliform glands are merely highly reduced (Fig. 2). Similar shifts in morphology have been observed in other spider lineages in which the building of orb webs has been modified or lost. Notably, in Cyrtophora (Araneidae), loss of the triad has been associated with the loss of sticky silk in the production of the mesh of dry silk [32]; in the species Drapetisca socialis and Neriene peltata (Linyphiidae), a similar loss is associated with the ability to catch prey outside the web without using glue in the web [33]; and in the subfamily Argyrodinae (Theridiidae), loss of the triad spigots is linked to abandonment of web building behavior and the adoption of kleptoparasitic or free-living existence [34]. Reduction in the triad is also known in the families Synaphridae [29] and Micropholcommatidae [44], though the predatory behavior in these lineages is not known. Among the Tetragnathidae, the genus Pachygnatha has largely lost the behavior of web building for prey capture and adults have lost the triad [26], although juvenile Pachygnatha still spin an orb web [45]. Apart from the Spiny Leg species examined in this study, the loss of the triad has also been documented in other Hawaiian tetragnathids. The monospecific Doryonychus raptor, which captures its prey without a foraging web, shows no 
Table 2 Results of COATS branch tests for positive selection

\begin{tabular}{|c|c|c|c|}
\hline Locus & Length & Best model & Annotation \\
\hline AM-TVBF-comp57563_c0_seq1.p1_RBHs & 93 & Non-web & $26 S$ proteasome non-ATPase regulatory subunit 14 (PSMD14) \\
\hline TVBF-comp44613_c0_seq1.p1_RBHs & 38 & Non-web & ankyrin repeat domain-containing protein 27-like \\
\hline AM-TVBF-comp45396_c0_seq1.p1_RBHs & 240 & Non-web & ATP synthase subunit b, mitochondrial \\
\hline AM-TVBF-comp58985_c0_seq2.p1_RBHs & 206 & Non-web & ATP synthase subunit beta, mitochondrial \\
\hline AM-TVBF-comp40348_c0_seq1.p1_RBHs & 88 & Non-web & Bystin-like (BYSL) \\
\hline AM-TVBF-comp56406_c0_seq1.p1_RBHs & 115 & Non-web & Cilia- and flagella-associated protein 20 \\
\hline AM-TVBF-comp20801_c0_seq1.p1_RBHs & 50 & Non-web & E3 ubiquitin-protein ligase (MARCH1) \\
\hline AM-TVBF-comp57815_c1_seq1.p1_RBHs & 94 & Non-web & E3 ubiquitin-protein ligase synoviolin (SYVN1) \\
\hline AM-TVBF-comp53073_c0_seq1.p1_RBHs & 101 & Non-web & Electron transfer flavoprotein subunit beta (ETFB) \\
\hline AM-TVBF-comp59149_c2_seq1.p1_RBHs & 160 & Non-web & MKI67 FHA domain-interacting nucleolar phosphoprotein-like \\
\hline AM-TVBF-comp56762_c0_seq1.p1_RBHs & 37 & Non-web & $\mathrm{N}$-alpha-acetyltransferase $35, \mathrm{NatC}$ auxiliary subunit \\
\hline AM-TVBF-comp49528_c2_seq1.p1_RBHs & 122 & Non-web & NEDD8-conjugating enzyme Ubc12 (UBE2M) \\
\hline AM-TVBF-comp26055_c0_seq1.p1_RBHs & 87 & Non-web & Obg-like ATPase 1 \\
\hline AM-TVBF-comp36800_c0_seq1.p1_RBHs & 138 & Non-web & Peroxisomal biogenesis factor 19 \\
\hline AM-TVBF-comp53850_c1_seq1.p1_RBHs & 91 & Non-web & PRKCA-binding protein \\
\hline TVBF-comp54710_c0_seq1.p1_RBHs & 162 & Non-web & Proteasome subunit alpha type-5 (PSMA5) \\
\hline TVBF-comp148043_c0_seq1.p1_RBHs & 149 & Non-web & Putative ATP-dependent RNA helicase me31b \\
\hline AM-TVBF-comp55490_c0_seq1.p1_RBHs & 66 & Non-web & Rab GDP dissociation inhibitor (GDI1) \\
\hline TVBF-comp57571_c0_seq1.p1_RBHs & 137 & Non-web & Ras-related protein Rab-5 \\
\hline AM-TVBF-comp38265_c0_seq1.p1_RBHs\ml & 102 & Non-web & Ras-related protein Rap-2c \\
\hline AM-TVBF-comp58242_c4_seq2.p1_RBHs & 203 & Non-web & Serine/threonine-protein kinase pim-3 \\
\hline AM-TVBF-comp56825_c0_seq1.p1_RBHs & 115 & Non-web & Synaptosomal-associated protein 29-like \\
\hline AM-TVBF-comp58649_c1_seq1.p1_RBHs & 115 & Non-web & Transitional endoplasmic reticulum ATPase TER94 \\
\hline AM-TVBF-comp49097_c0_seq2.p1_RBHs & 111 & Non-web & V-type proton ATPase subunit E \\
\hline AM-TVBF-comp40720_c0_seq1.p1_RBHs & 102 & Spiny Leg & 395 ribosomal protein L10, mitochondrial (MRPL10) \\
\hline AM-TVBF-comp57459_c0_seq1.p1_RBHs & 264 & Spiny Leg & $60 S$ acidic ribosomal protein PO (RPLP0) \\
\hline TVBF-comp58735_c0_seq1.p1_RBHs & 196 & Spiny Leg & DnaJ-like protein subfamily A member 1 \\
\hline TVBF-comp57965_c0_seq1.p1_RBHs & 344 & Spiny Leg & Elongation factor 2 (EEF2) \\
\hline TVBF-comp49143_c0_seq2.p1_RBHs & 131 & Spiny Leg & NADH dehydrogenase [ubiquinone] iron-sulfur protein 3, mitochondrial \\
\hline AM-TVBF-comp59471_c5_seq1.p1_RBHs & 128 & Spiny Leg & Splicing factor U2AF 50 kDa subunit \\
\hline TVBF-comp49479_c0_seq1.p1_RBHs & 158 & Spiny Leg & Synaptobrevin (YKT6) \\
\hline AM-TVBF-comp54231_c0_seq1.p1_RBHs & 162 & Spiny Leg & TATA-box-binding protein \\
\hline AM-TVBF-comp54631_c0_seq1.p1_RBHs & 72 & Spiny Leg & Ubiquitin-conjugating enzyme E2 Q1 (UBE2Q1) \\
\hline AM-TVBF-comp55223_c0_seq1.p1_RBHs & 115 & Spiny Leg & WD repeat-containing protein 44-like (WDR44) \\
\hline
\end{tabular}

Best model chosen by AIC. "Non-web" refers to a model in which selection acts on branches leading to "Spiny Leg" clade and to (Mimetidae + Malkaridae + Arkyidae). Spiny Leg refers to models where selection acts only on the Spiny Leg branch

evidence of the triad even during the earliest developmental stages [31]; prey are captured, either in flight or from the substrate, by impalement with the claws, which occurs through a very rapid movement of one or more of the first two pairs of legs [46].

\section{Changes in spidroin expression in Tetragnatha}

The transcriptomes of web-building Tetragnatha contain every major type of spidroin that has been described for the superfamily Araneoidea, while the Spiny Leg transcriptomes contain all major spidroins except for flagelliform (Fig. 3). We caution that absence of evidence is not evidence of absence. However, given the high completeness of our assemblies, the fact that flagelliform transcripts are absent from six separate Spiny Leg transcriptomes, and the absence of flagelliform spigots, we view it as likely that flagelliform spidroins are not expressed at appreciable levels. It remains to be seen whether flagelliform spidroins are present in Spiny Leg genomes, or have become pseudogenes due to loss of function and a release from selective restraints. In contrast, one species sequenced in this study (T. brevignatha) as well as three previously-sequenced Spiny Leg Tetragnatha [47] do express aggregate spidroins, despite 


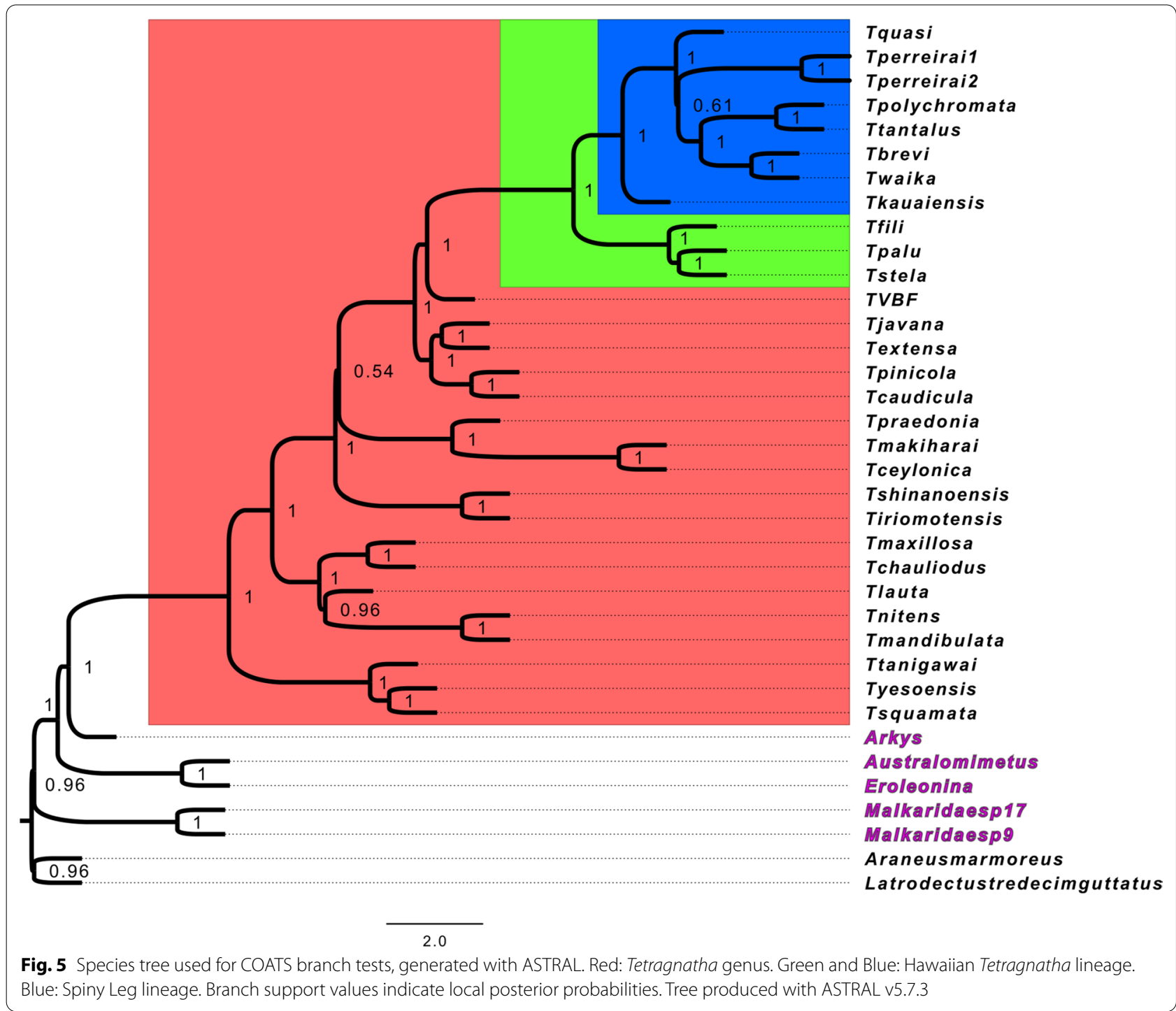

having lost aggregate silk glands (Fig. 3, Additional file 2). It is surprising that aggregate spidroins are expressed in species that cannot make aggregate silk. Spidroins are sometimes co-expressed in more than one silk gland [23, 48 ], and this could suggest that aggregate spidroins play a role in the production of other types of silk. Alternatively, it is likely that, because silk glands are derived from each other by serial homology, the ongoing transcription of aggregate spidroins is a remnant of past homology. This latter hypothesis is supported by the observation that aggregate transcripts in the Spiny Legs T. brevignatha and T. waikamoi were all expressed at quite low levels $(<1$ TPM), whereas web builders expressed aggregate spidroins at high levels (>100 TPM). Other studies have reported a similar phenomenon in male theridiid spiders, where loss of aggregate glands occurs upon reaching maturity, yet aggregate spidroins continue to be expressed [19]. Our selection analyses show that one aggregate spidroin appears to have experienced episodic diversifying selection in the Spiny Leg $T$. polychromata (Table 1), which suggests that it is performing some biological function. On the other hand, another aggregate spidroin has experienced relaxed selection in the Spiny Leg T. tantalus, which is consistent with a loss of function after the loss of aggregate silk production.

Spidroins generally fall into one of seven monophyletic groups corresponding to the seven silk glands [14]. However, the recent genome of the golden orb-weaver Trichonephila clavipes revealed several novel spidroins that do not fall into these well-established clades [14]. Our Tetragnatha transcriptomes contain homologs of some of these uncharacterized spidroins. Sp-5803 is primarily 
expressed in the flagelliform gland in T. clavipes $[14,40]$ and, like flagelliform spidroins, is absent from the Spiny Leg transcriptomes in this study, while being found in web builders (Fig. 3). Another uncharacterized spidroin, Sp-1339, shows evidence of relaxed selection in the entire Hawaiian Tetragnatha lineage (Table 1). A third spidroin, which forms a clade with T. clavipes Sp-907 and Sp-74867 (Fig. 3), has higher expression in Spiny Legs and has experienced relaxed selection both in Spiny Legs and in other non-web builders. We refer to this novel spidroin as Sp-907/Sp-74687. A previous study of major ampullate spidroins (MaSp) identified an expansion of MaSp-like sequences in Tetragnatha, including a "divergent spidroin variant" that lacked certain conserved residues [49]. These MaSp-like sequences (subtype F in [49]) are Sp-907/Sp-74687, having almost identical N-terminal domains (Additional file 4).

\section{Transcriptional shifts of silk gland-associated genes accompany loss of web-building}

Although spidroins make up the silk fibers themselves, many other genes play roles in silk production and have silk gland-specific expression. Previous studies have found that both peptidases and peptidase inhibitors are enriched among silk gland-specific genes. Clarke et al. suggest that this is because silk proteins that are successfully exported from cells need to be protected from degradation by peptidase inhibitors, whereas proteins that fail to properly assemble and export need to be degraded by peptidases [18]. Our results are consistent with this model, as both peptidases (e.g. a cathepisin and elastase) and peptidase inhibitors such as zonadhesins have shifted in expression between web builders and Spiny Legs. Interestingly, zonadhesins have been detected in the silk of the greater wax moth, which is not homologous to spider silk [50]. Silk gland-specific transcripts also include genes related to oxygen metabolism, at least in black widow spiders $[18,51]$. In the current study, we observe the downregulation of an amine oxidase and a monooxygenase in Spiny Legs, but the upregulation of a glutaredoxin and a dual oxidase. We also observed that Spiny Legs downregulate two genes that are highly expressed in the major ampullate glands of black widow spiders, a phosphoserine aminotransferase and a gamma-glutamyltransferase, the latter potentially involved in redox regulation in silk glands [48].

Genes with higher expression in Spiny Legs are enriched for functions related to chitin binding. While chitin serves primarily as a major structural component of arthropod bodies, forming the cuticle or exoskeleton, it is also found within the walls and ducts of silk glands, such as the major ampullate silk glands of Trichonephila clavipes and even the non-homologous silk glands of the silkworm [52]. Some spidroins also possess chitinbinding domains [53]. The close relationship between chitin and silk suggests that the changes in gene expression observed here may reflect morphological changes accompanying the loss of internal silk glands and external spigots. It is unclear why Spiny Legs have increased expression of chitin-binding genes. However, the loss of aggregate and flagelliform silk could be accompanied by increased expression of other types of silk such as dragline [19], leading to higher expression of some silkrelated genes.

Consistent with the loss of aggregate glands, Spiny Legs exhibited reduced expression of genes with aggregate gland-specific expression in other spiders, including a pp-GalNAc-T, a type of transferase that participates in $\mathrm{O}$-glycosylation [54]. O-glycosylation contributes to the adhesive qualities of aggregate glue [55], and pp-GalNAc$\mathrm{T}$ is highly expressed in the aggregate glands of the orbweaver Argiope trifasciata [41]. The reduced expression of this gene in Spiny Legs accompanied by their loss of aggregate glands suggests that this gene could play a similar role in aggregate silk production in tetragnathids. Spiny Legs also have reduced expression of a globin protein, a type of protein specifically expressed in the aggregate glands of Trichonephila clavipes [40]. Spiny Legs have also downregulated other genes identified in the aggregate and flagelliform glands of Trichonephila clavipes [40], including an aminopeptidase, a serine/threonine protein kinase, and an acyl-CoA dehydrogenase. However, further work is needed to understand gland-specific patterns of gene expression in Tetragnatha.

\section{Convergent web loss is associated with selection on protein and energy metabolism}

Branch tests of single-copy orthologs identified targets of selection along branches where independent web loss events have occurred in Tetragnatha and related lineages (species shown in Fig. 5). Many of the same genes, including ubiquitin ligases and proteasome components, appear to be targets of selection in both Spiny Legs and other non-web building families (Table 2). Other genes in this category include pim-3, a kinase that regulates protein synthesis [56], and BYSL, which mediates ribosome biogenesis [57]. This suggests that the loss of web silk is accompanied by convergent selection on protein synthesis, processing, and degradation machinery in both Spiny Leg Tetragnatha and other non-web building araneoid spiders. Since silk is a proteinaceous material, changes in the silk needs of a species may require changes in genes regulating protein metabolism. There is an interesting parallel in silkworms, where QTL analysis identified polymorphism in a ribosomal subunit associated with silk synthesis [58]. Another interesting category 
of genes with convergent selection is related to energy metabolism, including both ATP synthase and ATPases. There may be an association between large-scale changes in silk production and changes in cellular metabolism. Other genes, including ribosomal components and genes related to vesicle transport, are targets of selection only in Spiny Leg Tetragnatha, suggesting that lineage-specific differences are also important. Still, it is notable that even in cases when selection has not acted on the same gene, it acts on genes with functions related to protein metabolism.

\section{Role for a novel spidroin in web loss}

We have identified an as-yet uncharacterized spidroin, Sp-907/Sp-74867, that shows evidence for convergent relaxed selection in Spiny Leg Tetragnatha and the nonweb builders Malkaridae sp. 17 and Australomimetus sp.; this gene has also experienced episodic diversifying selection in Malkaridae sp. 17 (Table 1). Relaxed selection can be caused not only by loss of function, but also by a change in functional constraints upon adaptation to a new ecological niche, or a reduction in effective population size [59]. Since we also observed increased expression of Sp-907/Sp-74867 in Spiny Legs, we suggest a role for this spidroin in the transition to a cursorial lifestyle. The function of Sp-907/Sp-74867 is unclear; however, in Trichonephila clavipes, expression of Sp-74867 correlates with major ampullate (dragline) spidroin expression [14]. Correa-Garwhal et al. found that male Steatoda grossa, which are cursorial, have higher expression of major ampullate spidroins than females, and suggest that major ampullate silk has an increased or different role in cursorial males that could be due to differences in dragline silk [19]. Sp-907/Sp-74867 expression might also be related to changes in dragline silk related to the transition to cursoriality. Future work localizing Sp-907/Sp-74867 to particular silk glands would help clarify its function, and it may also be interesting to examine differences in the structural composition of silks from web building and non-web building taxa.

It is important to note that the genes identified in this study are not exhaustive. Our gene expression analysis is limited by small sample sizes, meaning that we only have power to detect the largest changes in expression. Moreover, RNA-seq data can inflate expression estimates of spidroins when reads derived from repetitive regions map to fragments containing portions of the repetitive region [60]. Our selection analyses are constrained by the relative incompleteness of some transcriptomes, which limits the number of single-copy orthologs that we can recover. The transcriptomes sequenced in this study are quite complete, but other assemblies were generated with different goals where completeness was less important [47, 61]. This type of analysis also neglects the roles of gene loss and duplication in character evolution. Finally, transcriptomic data are rarely able to reconstruct full-length spidroins, which means that we cannot match $\mathrm{N}$ - and $\mathrm{C}$-terminal sequences. The sequences in our phylogenetic and selection analyses correspond to internal, $\mathrm{N}$-, or C-terminal fragments of full-length spidroins, which may have experienced different selection regimes. Nonetheless, our complementary analyses provide a clearer picture of the types of genes involved in web loss and of the distribution of spidroins in non-web building Tetragnatha. In particular, protein synthesis and degradation machinery appear to be convergent targets of selection when capture webs are lost.

\section{Future directions}

The loss of web building behavior has occurred independently at many different levels: within the genus Tetragnatha, between genera within the Tetragnathidae, and between the related families shown in this study. This system therefore provides an interesting opportunity to compare genomic shifts across phylogenetic scales. Intriguingly, web loss within Tetragnatha is not only associated with loss of the aggregate/flagelliform triad, but also with a marked ecological shift into forest habitats, the display of bright green coloration with red color polymorphism, and the development of greatly elongated macrosetae ("spines") on the forelegs [46]. The American T. viridis is a bright green forest dweller that has abandoned web building and has elongated spines on the legs [62]; T. laqueata from the Bonin Islands is also a spiny green forest dweller, as are T. squamata from Japan, $T$. tanigawai from the Ryukus [63], and T. viridorufa from India [64]. Future work comparing the genomic basis for these suites of convergent traits will provide insights into the interplay between genotype and phenotype that allows these characters to evolve together.

\section{Conclusions}

Phenotypic convergence has been broadly defined as the independent evolution of similar phenotypes. There are numerous examples of convergence, though only a small fraction of studies have examined whether convergent events are based on shared genetic mechanisms [12]. Our study is the first to systematically characterize silk genes in Spiny Leg Tetragnatha, and determine associations with convergent adaptation of a cursorial lifestyle. Web loss is associated with a series of morphological, ecological, and behavioral traits, and we show that web loss is accompanied by shifts in the expression of silk-associated genes within Tetragnatha. Our results further show that selection has acted on many of the same protein-coding 
genes in independent lineages that have lost webs and shifted to a cursorial lifestyle.

\section{Methods \\ Morphology of spinneret spigots and silk glands}

Scanning electron microscopy was conducted on the spinnerets of single individual females of seven web spinners: Tetragnatha acuta, T. trituberculata, T. hawaiensis, T. eurychasma, T. sp. "Little wave", T. stelarobusta, and T. sp. "Long-clawed legs"; and four species in the Spiny Leg clade: T. kauaiensis, T. quasimodo (two individuals), T. macracantha, and T. waikamoi (two individuals). These are shown in Fig. 1. Specimens were imaged either in 1990 or 2016, following slightly different protocols. In the early study (1990), live spiders were boiled in water for $40 \mathrm{~s}$ in order to cause the spinneret spigots to evert. In more recent work (2016), dead specimens preserved in $70 \%$ ethanol were used; spinnerets were everted by pinching the abdominal tissue at their base with finetipped forceps. The spinnerets were then cut from the body and placed in plastic capsules with the central portion removed and nylon mesh placed inside the capsule (to allow exchange of alcohol and $\mathrm{CO}_{2}$, while retaining the specimen). Filled capsules were put through an alcohol series $(70 \%, 85 \%, 95 \%$ and pure ethanol), then dried with an Autosamori-810 (in 1990) or a Bal-Tec CPD 030 (in 2016) critical point dryer. The structures were then removed from the capsules, mounted on stubs and sputtered with gold, and then viewed using a Hitachi S-800 (in 1990) or a Philips XL-30 ESEM (in 2016) scanning electron microscope.

\section{Animal collection for RNA extraction}

We collected six species of Tetragnatha on Maui in June and September 2017: the web-builders T. stelarobusta, T. filiciphilia, and T. paludicola, and the Spiny Legs T. waikamoi, T. brevignatha, and T. quasimodo. We sequenced total RNA from two individuals of each species, for a total of twelve sequence libraries. All individuals were females and sexually mature at the time of extraction. The spiders were kept in the lab in Berkeley, California until they were extracted in November and December of that year. Animals were maintained under a common temperature, humidity, and light regime and fed at the same time each day. Extractions were performed on two days, at the same time each day. Live spiders were anesthetized with carbon dioxide and their abdomens were severed at the narrowest point of connection with the cephalothorax. Silk glands were dissected out as one mass in the presence of SSC buffer and preserved in RNAlater (procedure adapted from [65]). Due to the small size of the abdomens (approximately $5 \mathrm{~mm}$ in length), not all skin and abdominal tissue was excised. During dissections, each species' complement of silk glands was observed. Excised tissues were homogenized and RNA extraction was performed using a Qiagen RNeasy Extraction Kit following the manufacturer's protocol. RNA concentration and quality were verified for each sample using a Qubit fluorometer and a NanoDrop spectrophotometer. We also included a set of Tetragnatha spiders that were collected from different areas in Japan. These samples were all brought back to the lab alive (in less than a week), and were snap frozen with liquid nitrogen, then stored at $-80^{\circ} \mathrm{C}$ until they were subjected to RNA extraction. For collecting information and accession numbers, see Additional file 5 .

\section{Library construction and sequencing}

Library preparation was performed using a KAPA RNA Library Preparation Kit, following the manufacturer's protocol. To summarize, poly-A mRNA selection was performed using magnetic oligo-dT beads. RNA was fragmented to the desired size (approx. 350-450 bp) by heating to $85{ }^{\circ} \mathrm{C}$ in fragmentation buffer. First-strand cDNA was synthesized with random primers, and second-strand cDNA was synthesized and marked with dUTP to preserve strandedness. Double-stranded cDNA libraries were A-tailed and ligated to dual-index Illumina adapters. The adapter-ligated libraries were amplified with 10 PCR cycles. Finally, library concentration and fragment size were assessed with a Bioanalyzer and Qubit fluorometer. Paired-end sequencing was performed on an Illumina HiSeq 4000 by the Vincent J. Coates Genomics Sequencing Laboratory at UC Berkeley. Sequencing for Japanese Tetragnatha (unpublished data) was performed as described previously in [66].

\section{De novo transcriptome assembly and filtering}

Quality filtering was conducted with Trimmomatic v0.38 [67] using conservative parameters (LEADING:5 TRAILING:5 SLIDINGWINDOW:5:5 MINLEN:50). The six species transcriptomes were each assembled using Trinity v2.4.0 [68], with default parameters except for omitting in silico normalization (-no-normalize-reads). To evaluate assembly quality, we mapped trimmed reads back to their assembly using Bowtie v2.3.4 [69]. Assembly completeness was assessed with the arthropod set of BUSCO (Benchmarking Universal Single Copy Orthologs) v3.0.2 [70] (downloaded February 7, 2017).

\section{Protein prediction and annotation}

We used TransDecoder v5.3.0 (http://transdecoder. github.io) to generate predicted open reading frames (ORF's), with a minimum length of 100 amino acids. We subjected these candidate peptides to three homology 
searches: using DIAMOND v0.9.22.123 [71] (with the '-more-sensitive' flag), we searched against NCBI's nonredundant nr protein database (downloaded on March 23,2019 ), and against a custom database of $\mathrm{N}$ - and $\mathrm{C}$-terminal spidroin sequences from unpublished Tetragnatha transcriptomes. We also used hmmscan v3.1b2 (available from hmmer.org) to search the Pfam protein database (downloaded February 22, 2019). TransDecoder retained the single best ORF for each transcript, and any predicted peptides with an e-value of less than 1e-10 against any database were also retained in the final set. We then used CD-HIT v4.7 [72] to merge peptides with $100 \%$ identity, to produce six non-redundant protein-coding transcriptomes. We also annotated single-copy orthologs used in the differential expression analysis (see below) with BLASTP v2.10.0 against the $\mathrm{nr}$ database (downloaded November 23, 2020), with an e-value cutoff of $1 \mathrm{e}-10$, and these annotations are reported in Additional file 3.

\section{Orthogroup assignment}

We used the program OrthoFinder v2.2.7 [73] to assign proteins to orthogroups and construct a rooted gene tree for each group. An orthogroup is a monophyletic group of genes descended from an ancestral gene in the last common ancestor of the species being analyzed, and can include both orthologs and paralogs. OrthoFinder also infers a species tree from the single-copy gene trees. We then used PhyloTreePruner v1.0 [74] to define a set of single-copy orthologs with one representative in each species. For gene trees with monophyletic species-specific gene duplications (in-paralogs), PhyloTreePruner retains only the longest paralog sequence. This approach is particularly relevant for transcriptomic data, as apparent in-paralogs are often alternative splice variants of the same gene.

\section{Transcript quantification and differential expression}

Transcript abundance for each library was quantified using Salmon v0.13.1 [75]. Indices were constructed for the six transcriptomes with a k-mer size of 31, and mapping was performed with the following parameters: 'validateMappings -rangeFactorizationBins 4 -seqBias -gcBias.' We used the R package tximport v1.10.1 [76] to summarize transcript-level abundance to the orthogroup/gene level. In order to combine the data from different transcriptomes, we edited transcript names to append a species identifier to the Trinity contig name. For each library, we then added the transcript names for the other five species, with a count of zero. For example, a T. waikamoi counts file would include its count data for the T. waikamoi transcripts, and zero counts for all transcripts from the other five species. We provided a file relating transcript ID's and single-copy orthogroups. This means that only transcripts belonging to one of the single-copy orthologs were retained for differential expression analysis, although reads were originally mapped to the entire transcriptome. Importantly, tximport corrects for differences in transcript length when summarizing to the gene level.

We used the R package DESeq2 v1.22.2 [77] to test for differential expression between the web building and Spiny Leg groups. The six individuals within each group (belonging to three species) were treated as replicates. We were able to compare gene expression across species due to the use of common garden conditions and use of a single life stage. Genes (single-copy orthologs) with logfold change greater than or equal to 0.5 at an FDR of 0.05 were considered differentially expressed. We also estimated shrunken log-fold changes using the 'IfcShrink()' function with the method 'apeglm' and 'IfcThreshold $=0.5^{\prime}$, and used these shrunken log-fold changes for gene ontology enrichment. The code for this analysis is available in Additional file 6.

\section{Gene ontology enrichment analysis}

We retrieved GO annotations using Blast2GO v5.2.5 [78] on the output of the DIAMOND search against nr (with an e-value cutoff of 1e-10). This was done for each transcriptome, and orthogroups were annotated by combining the GO terms from all transcripts belonging to that orthogroup. Of the 7835 single-copy orthologs, 6648 received at least one $\mathrm{GO}$ annotation. GO enrichment was analyzed using the R package GO_MWU [79] on the shrunken log-fold changes, with default settings.

\section{Spidroin phylogeny}

We aligned $\mathrm{N}$ - and $\mathrm{C}$-terminal domain amino acid sequences with the M-Coffee web server [80]. Bayesian majority consensus trees were constructed using $\mathrm{Mr}$. Bayes v3.2.7 [81] with a mixed amino acid model and a gamma rate distribution. Monte Carlo Markov Chain sampling was run for 5 million generations (or 1 million for the smaller aggregate spidroin tree), with four chains and a relative burn-in of $25 \%$, for two independent runs. Trees were visualized on the iTOL web server [82] and rooted with a mygalomorph spidroin (B. californicum fibroin), or with flagelliform spidroins in the case of the aggregate spidroin tree.

\section{Selection analyses}

These analyses set out to look at signatures of selection operating on the different silks. In particular, we tested for diversifying and relaxed selection associated with shifts from web building to cursorial lifestyle and with the loss of web building behavior. Moreover, we tested to see whether similar patterns of selection operated in 
different lineages where web loss has been documented. We included other published (from $[47,83]$ ) and unpublished Tetragnatha transcriptomes, as well as transcriptomes from three other non-web building families (Arkyidae, Malkaridae, and Mimetidae) and two Araneoid web-building outgroups in the analyses (from [61]). We used several complementary approaches to test for selection in silk genes. First, we used a gene family clustering approach implemented in the FUSTr pipeline (Families Under Selection in Transcriptomes [42]), which clusters sequences from all available transcriptomes into putative gene families. These gene families do not necessarily contain sequences from all taxa, and given the structure of spidroins (discussed in the introduction) are likely to contain only fragments of a larger gene. As input, we used our six new Tetragnatha transcriptomes, 23 additional Tetragnatha transcriptomes corresponding to 22 species (T. perreirai represented twice) $[47,83]$, five non-web building spiders from other families from [61] (Arkys sp., Australomimetus sp., Ero leonina, Malkaridae sp. 9, Malkaridae sp. 17), and two araneoid web building outgroup species from the same study (Araneus marmoreus, Latrodectus tredecimguttatus), for a total of 36 transcriptomes/35 species. FUStr translates sequences and predicts open reading frames using TransDecoder, assigns genes to homologous families using BLASTP and the clustering algorithm SiLix (v1.2.11) [84], generates multiple sequence alignments with MAFFT [85], and builds trees for each family using FastTree [86]. For each gene family with at least 15 sequences, we then conducted site tests for positive selection using FUBAR [87]. For gene families with BLAST hits to Trichonpehila clavipes spidroin sequences, we implemented branch tests using aBSREL [88] and RELAX [59], which test for diversifying and relaxed selection, respectively. FUBAR, aBSREL, and RELAX were implemented in HyPhy v2.5.14.

An additional strategy was used to test for selection in single-copy orthologs in the context of the species tree, using the COATS pipeline (unpublished, Brewer et al. in prep, https://hub.docker.com $/ \mathrm{r} /$ michaelsbrewer/coats_ test). Unlike FUSTr, COATS only considers single-copy orthologs present in all taxa, with histories that are consistent with the species tree. The species tree was generated from unrooted gene trees using ASTRAL v5.7.3 [89]. COATS generates orthologous gene sets using reciprocal best hits with BLASTP, which are then aligned with MAFFT [85] and codon alignments are generated with $\mathrm{Pal} 2 \mathrm{Nal}$ [90]. Branch tests were then conducted using the codeml module of PAML [91]. We limited our analysis to orthologs consistent with the species tree, as evaluated with the approximately unbiased test in IQTREE v2.0 [92]. None of these single-copy orthologs were spidroins, likely because of the relative incompleteness of many of the transcriptomes included in the analysis. We tested selection in two scenarios, one in which selection acts on the branch leading to the Spiny Leg clade, and another in which selection acts on the branches leading to Spiny Legs and to the other non-web builders (essentially, testing for convergence).

\begin{abstract}
Abbreviations
AcSp: Aciniform spidroin; AgSp: Aggregate spidroin; ALS: Anterior lateral spinnerets; ATP: Adenosine triphosphate; bp: Base pair; BUSCO: Benchmarking universal single copy orthologs; BYSL: Bystin-like; CDNA: Complementary deoxyribonucleic acid; C-terminus: Carboxyl terminus; DE: Differentially expressed; dUTP: Deoxyuridine triphosphate; FDR: False discovery rate; Flag: Flagelliform spidroin; GO: Gene ontology; MaSp: Major ampullate spidroin; MiSp: Minor ampullate spidroin; mya: Million years ago; NCBl: National Center for Biotechnology Information; N-terminus: Amino terminus; ORF: Open reading frame; PCR: Polymerase chain reaction; PiSp: Piriform spidroin; PLS: Posterior lateral spinnerets; PMS: Posterior medial spinnerets; pp-GalNAc-T: N-acetylgalactosaminyltransferase; QTL: Quantitative trait loci; RNA: Ribonucleic acid; SSC: Saline-sodium citrate; TPM: Transcripts per million; TuSp: Tubuliform spidroin.
\end{abstract}

\section{Supplementary Information}

The online version contains supplementary material available at https://doi. org/10.1186/s12862-021-01779-9.

Additional file 1. Read and assembly statistics. a: Percent of trimmed reads mapping as proper pairs to the assembly using Bowtie2. b: Peptides predicted by TransDecoder. c: Peptides after clustering at 100\% identity with CD-HIT.

Additional file 2. Bayesian consensus tree of aggregate spidroin C-terminal domains. Branch labels show posterior probabilities. Branches leading to species sequenced in this study are colored red, and Spiny Leg species are labelled with blue text. Tree produced using Mr. Bayes v3.2.7 and visualized with iTOL web server.

Additional file 3. List of differentially expressed genes and annotations. Includes top 3 BLASTP hits against NCBI's nonredundant nr database (accessed November 23, 2020).

Additional file 4. N-terminal amino acid alignment of Sp-907/Sp-74867 sequences from Malay et al. [77]. The N-terminal domains of "MaSp-f" sequences in [77] are almost identical to Sp-907/Sp-74867, and differ from those of other MaSp sequences. Alignment generated with M-Coffee.

Additional file 5. Sample information. Includes collection information (locale and date) for individuals sequenced in this study, as well as GenBank accession numbers.

Additional file 6. $R$ code used for differential expression analysis.

\section{Acknowledgements}

The permit processing and access to different reserves and private land was possible thanks to Steve Bergfeld (DOFAW Big Island), Pat Bily (TNC Maui), Tabetha Block (HETF), Lance DaSilva (DOFAW Maui), Charmian Dang (NAR), Melissa Dean (HETF), Betsy Gagne (NAR), Elizabeth Gordon (HALE), Cynthia King (DLNR), Russell Kallstrom (TNC Moloka ‘i), Joey Mello (DOFAW Big Island), Ed Misaki (TNC Moloka'i), and Kawika Winter (Limahuli Botanical Garden). Imaging for the spinnerets was supported by an NSF Graduate Research Internship Program (GRIP) to SRK, with thanks to Scott Whittaker at the National Museum of Natural History Imaging Lab at the Smithsonian Institution. For the earlier imaging, RGG thanks the Zoology Department, U.H. Manoa, and in particular Marilyn Dunlap and Tina Carvalho. We thank Anna Wood and Gregg Stephenson for help with collecting (funded by the UC Berkeley Sponsored Projects for Undergraduate Research). We are also grateful 
to Jessica Garb and Cheryl Hayashi for their suggestions regarding dissection of silk glands.

\section{Authors' contributions}

$C A B, S A A$, and RGG conceived of and designed the study. CAB and SAA performed lab work. CAB dissected silk glands, assembled transcriptomes, and conducted gene expression and phylogenetic analyses. SAA collected animals and edited the manuscript. NK, HN, and KA sequenced and assembled transcriptomes used in selection and phylogenetic analyses. SRK and HMW performed microscopy and edited the manuscript. CAB, SAA, and SRK designed figures. MSB performed the selection analyses and contributed to the interpretation of results. CAB and RGG wrote the manuscript. All authors read and approved the final manuscript.

\section{Funding}

Funding for the study was provided by a grant from UC Berkeley's Summer Undergraduate Research Fellowship (SURF) to CAB, with additional support from an NSF Graduate Research Fellowship (SAA), and the Schlinger Foundation (RGG). The funding bodies played no role in the design of the study and collection, analysis, and interpretation of data and in writing the manuscript.

\section{Availability of data and materials}

The datasets supporting the conclusions of this article are available in the project GitLab repository, https://gitlab.com/Brevver/tetragnathasilks. Sequenced reads and assemblies are available on the Short Read Archive and the Transcriptome Shotgun Assembly (TSA) database, respectively (BioProject PRJNA650562). The TSA projects have been deposited at GenBank under the accessions GITM00000000, GITN00000000, GITO00000000, GITP00000000, GITQ00000000, and GITR00000000. The versions described in this paper are the first versions, numbered GITM01000000 and so on. All sample information and accession numbers are given in Additional file 5.

\section{Declarations}

\section{Ethics approval and consent to participate}

Spiders were collected under State of Hawai'i Department of Land and Natural Resources Scientific Permit No. 11026.

\section{Consent for publication}

Not applicable.

\section{Competing interests}

The authors declare no competing interests.

\begin{abstract}
Author details
${ }_{1}^{1}$ Department of Environmental Science, Policy and Management, University of California, Berkeley, 130 Mulford Hall, \#3114, Berkeley, CA 94720-3114, USA. 2 Present Address: MIT-WHOI Joint Program in Oceanography/Applied Ocean Science and Engineering, Cambridge, Woods Hole, MA, USA. ${ }^{3}$ Department of Biology, N1088 Howell Science Complex, East Carolina University, Greenville, NC 27858, USA. ${ }^{4}$ Institute for Advanced Biosciences, Keio University, Yamagata, Japan. ${ }^{5}$ Enzyme Research Team, RIKEN Center for Sustainable Resource Science, 2-1 Hirosawa, Wako-shi, Saitama 351-0198, Japan. ${ }^{6}$ Biodiversity and Biocomplexity Unit, Okinawa Institute of Science and Technology Graduate University, Tancha 1919-1, Onna, Okinawa 904-0495, Japan. ${ }^{7}$ Smithsonian Institution, Entomology, MRC105, Natural History Bldg. E519, 1000 Constitution Ave NW, Washington DC 20560-0188, USA.
\end{abstract}

Received: 21 August 2020 Accepted: 10 March 2021 Published online: 22 March 2021

\section{References}

1. Wake DB, Wake MH, Specht CD. Homoplasy: from detecting pattern to determining process and mechanism of evolution. Science. 2011;331:1032-5.

2. Losos J. Lizards in an evolutionary tree: ecology and adaptive radiation of anoles. Berkeley: University of California Press; 2009.
3. Gillespie R. Community assembly through adaptive radiation in Hawaiian spiders. Science. 2004;303:356-9.

4. Springer M, Kirsch J, Case J. The chronicle of marsupial evolution. In: Givnish TJ, Sytsma KJ, eds. Molecular evolution and adaptive eadiation; 1997. pp. 129-161.

5. Chikina M, Robinson JD, Clark NL. Hundreds of genes experienced convergent shifts in selective pressure in marine mammals. Mol Biol Evol. 2016;33:2182-92

6. Natarajan C, Projecto-Garcia J, Moriyama H, Weber RE, Muñoz-Fuentes V, Green AJ, et al. Convergent evolution of hemoglobin function in highaltitude Andean waterfowl involves limited parallelism at the molecular sequence level. PLOS Genet. 2015;11:e1005681.

7. Woodard SH, Fischman BJ, Venkat A, Hudson ME, Varala K, Cameron SA, et al. Genes involved in convergent evolution of eusociality in bees. Proc Natl Acad Sci U S A. 2011;108:7472-7.

8. Parker J, Tsagkogeorga G, Cotton JA, Liu Y, Provero P, Stupka E, et al. Genome-wide signatures of convergent evolution in echolocating mammals. Nature. 2013;502:228-31.

9. Sackton TB, Grayson P, Cloutier A, Hu Z, Liu JS, Wheeler NE, et al. Convergent regulatory evolution and loss of flight in paleognathous birds. Science. 2019;364:74-8.

10. Jeffery WR. Regressive evolution in Astyanax cavefish. Annu Rev Genet. 2009;43:25-47.

11. Protas M, Conrad M, Gross JB, Tabin C, Borowsky R. Regressive evolution in the Mexican cave tetra, Astyanax mexicanus. Curr Biol CB. 2007;17:452-4.

12. Rosenblum EB, Parent CE, Brandt EE. The molecular basis of phenotypic convergence. Annu Rev Ecol Evol Syst. 2014;45:203-26.

13. Elmer KR, Meyer A. Adaptation in the age of ecological genomics: insights from parallelism and convergence. Trends Ecol Evol. 2011;26:298-306.

14. Babb PL, Lahens NF, Correa-Garhwal SM, Nicholson DN, Kim EJ, Hogenesch JB, et al. The Nephila clavipes genome highlights the diversity of spider silk genes and their complex expression. Nat Genet. 2017;49:895-903.

15. Coddington JA, Agnarsson I, Hamilton CA, Bond JE. Spiders did not repeatedly gain, but repeatedly lost, foraging webs. PeerJ. 2019;7:e6703.

16. Guerette PA, Ginzinger DG, Weber BHF, Gosline JM. Silk properties determined by gland-specific expression of a spider fibroin gene family. Science. 1996;272:112-5.

17. Gatesy J, Hayashi C, Motriuk D, Woods J, Lewis R. Extreme dversity, conservation, and convergence of spider silk fibroin sequences. Science. 2001;291:2603-5.

18. Clarke TH, Garb JE, Hayashi CY, Haney RA, Lancaster AK, Corbett S, et al. Multi-tissue transcriptomics of the black widow spider reveals expansions, co-options, and functional processes of the silk gland gene toolkit. BMC Genomics. 2014;15:365.

19. Correa-Garhwal SM, Chaw RC, Clarke TH, Ayoub NA, Hayashi CY. Silk gene expression of theridiid spiders: implications for male-specific silk use. Zoology. 2017;122:107-14.

20. Garb JE, Ayoub NA, Hayashi CY. Untangling spider silk evolution with spidroin terminal domains. BMC Evol Biol. 2010;10:243.

21. Starrett J, Garb JE, Kuelbs A, Azubuike UO, Hayashi CY. Early events in the evolution of spider silk genes. PLoS ONE. 2012;7:e38084.

22. Clarke TH, Garb JE, Hayashi CY, Arensburger P, Ayoub NA. Spider transcriptomes identify ancient large-scale gene duplication event potentially important in silk gland evolution. Genome Biol Evol. 2015;7:1856-70.

23. Clarke TH, Garb JE, Haney RA, Chaw RC, Hayashi CY, Ayoub NA. Evolutionary shifts in gene expression decoupled from gene duplication across functionally distinct spider silk glands. Sci Rep. 2017;7:8393.

24. Kovoor J. Comparative structure and histochemistry of silk-producing organs in arachnids. In: Nentwig W, ed. Ecophysiology of spiders. Berlin Heidelberg: Springer; 1987. p. 160-86. https://doi.org/10.1007/978-3-64271552-5_12.

25. Coddington JA. Spinneret silk spigot morphology: Evidence for the monophyly of orbweaving spiders, Cyrtophorinae (Araneidae), and the group Theridiidae plus Nesticidae. J Arachnol. 1989;17:71-95.

26. Griswold CE, Coddington JA, Hormiga G, Scharff N. Phylogeny of the orbweb building spiders (Araneae, Orbiculariae: Deinopoidea, Araneoidea). Zool J Linn Soc. 1998;123:1-99.

27. Coddington J. The monophyletic origin of the orb web. 1986;54.

28. Kovoor J, Zylberberg L. Ultrastructure du canal des glandes agrégées et flagelliformes d'Araneus diadematus Clerck (Araneae, Araneidae). Zoomorphologie. 1979;92:217. 
29. Miller JA. Synaphridae of Madagascar (Araneae: Araneoidea): a new family record for the Afrotropical region. Proc Calif Acad Sci. 2007;58:29.

30. Townley MA, Tillinghast EK. Aggregate silk gland secretions of araneoid spiders. In: Nentwig W, editor. Spider ecophysiology. Berlin, Heidelberg: Springer; 2013. p. 283-302. doi:https://doi.org/10.1007/978-3-642-339899_21.

31. Gillespie R. Impaled prey. Nature. 1992;355:212-3.

32. Peters HM. Functional organization of the spinning apparatus of Cyrtophora citricola with regard to the evolution of the web (Araneae, Araneidae). Zoomorphology. 1993;113:153-63.

33. Schutt K. Drapetisca socialis (Araneae: Linyphiidae): Web reductionethological and morphological adaptations. EJE. 1995;92:553-63.

34. Agnarsson I. Morphological phylogeny of cobweb spiders and their relatives (Araneae, Araneoidea, Theridiidae). Zool J Linn Soc. 2004;141:447-626.

35. Benavides LR, Hormiga G. A morphological and combined phylogenetic analysis of pirate spiders (Araneae, Mimetidae): evolutionary relationships, taxonomy and character evolution. Invertebr Syst. 2020;34:144-91.

36. Gillespie RG. Island time and the interplay between ecology and evolution in species diversification. Evol Appl. 2016:9:53-73.

37. Gillespie RG. Hawaiian spiders of the genus Tetragnatha: I. Spiny leg clade J Arachnol. 1991;19:174-209.

38. Whaite AD, Wang T, Macdonald J, Cummins SF. Major ampullate silk gland transcriptomes and fibre proteomes of the golden orb-weavers, Nephila plumipes and Nephila pilipes (Araneae: Nephilidae). PLoS ONE. 2018;13:e0204243.

39. Ayoub NA, Hayashi CY. Multiple recombining loci encode MaSp1, the primary constituent of dragline silk, in widow spiders (Latrodectus: Theridiidae). Mol Biol Evol. 2008;25:277-86.

40. dos Santos-Pinto JRA, Esteves FG, Sialana FJ, Ferro M, Smidak R, Rares LC, et al. A proteotranscriptomic study of silk-producing glands from the orbweaving spiders. Mol Omics. 2019;15:256-70.

41. Stellwagen SD, Renberg RL. Toward spider glue: Long read scaffolding for extreme length and repetitious silk family genes AgSp1 and AgSp2 with insights into functional adaptation. G3 Genes Genomes Genet. 2019;9:1909-19.

42. Cole TJ, Brewer MS. FUSTr: a tool to find gene families under selection in transcriptomes. PeerJ. 2018;6:e4234

43. Alfaro RE, Griswold CE, Miller KB. Comparative spigot ontogeny across the spider tree of life. PeerJ. 2018:6:e4233.

44. Rix MG, Harvey MS. The spider family Micropholcommatidae (Arachnida: Araneae: Araneoidea): a relimitation and revision at the generic level. Bulgaria: PenSoft Publishers LTD; 2010.

45. Platnick NI, Coddington JA, Forster RR, Griswold CE. Spinneret morphology and the phylogeny of haplogyne spiders (Araneae, Araneomorphae). American Museum Novitates. 1991.

46. Gillespie RG. Predation through impalement of prey: The foraging behavior of Doryonychus raptor (Araneae: Tetragnathidae). Psyche (Stuttg). 1991:98:337-50.

47. Brewer MS, Carter RA, Croucher PJP, Gillespie RG. Shifting habitats, morphology, and selective pressures: developmental polyphenism in an adaptive radiation of Hawaiian spiders. Evolution. 2014;69:162-78.

48. Lane AK, Hayashi CY, Whitworth GB, Ayoub NA. Complex gene expression in the dragline silk producing glands of the Western black widow (Latrodectus hesperus). BMC Genomics. 2013;14:1-12.

49. Malay AD, Arakawa K, Numata K. Analysis of repetitive amino acid motifs reveals the essential features of spider dragline silk proteins. PLOS ONE. 2017:12:e0183397.

50. Kludkiewicz B, Kucerova L, Konikova T, Strnad H, Hradilova M, Zaloudikova $A$, et al. The expansion of genes encoding soluble silk components in the greater wax moth, Galleria mellonella. Insect Biochem Mol Biol. 2019;106:28-38

51. Chaw RC, Correa-Garhwal SM, Clarke TH, Ayoub NA, Hayashi CY. Proteomic evidence for components of spider silk synthesis from black widow silk glands and fibers. J Proteome Res. 2015;14:4223-31.

52. Davies GJG, Knight DP, Vollrath F. Chitin in the silk gland ducts of the spider Nephila edulisand the silkwormBombyx mori. PLOS ONE. 2013. https:// doi.org/10.1371/journal.pone.0073225.

53. Collin MA, Clarke TH, Ayoub NA, Hayashi CY. Evidence from multiple species that spider silk glue component ASG2 is a spidroin. Sci Rep. 2016:6:21589.
54. Wang H, Tachibana K, Zhang Y, Iwasaki H, Kameyama A, Cheng L, et al. Cloning and characterization of a novel UDP-GalNAc:polypeptide $\mathrm{N}$-acetylgalactosaminyltransferase, pp-GalNAc-T14. Biochem Biophys Res Commun. 2003;300:738-44.

55. Singla S, Amarpuri G, Dhopatkar N, Blackledge TA, Dhinojwala A. Hygroscopic compounds in spider aggregate glue remove interfacial water to maintain adhesion in humid conditions. Nat Commun. 2018;9:1-8.

56. Beharry Z, Mahajan S, Zemskova M, Lin Y-W, Tholanikunnel BG, Xia Z, et al. The Pim protein kinases regulate energy metabolism and cell growth. Proc Natl Acad Sci. 2011;108:528-33.

57. Fukuda MN, Miyoshi M, Nadano D. The role of bystin in embryo implantation and in ribosomal biogenesis. Cell Mol Life Sci. 2008;65:92-9.

58. Li C, Tong X, Zuo W, Luan Y, Gao R, Han M, et al. QTL analysis of cocoon shell weight identifies BmRPL18 associated with silk protein synthesis in silkworm by pooling sequencing. Sci Rep. 2017;7:17985.

59. Wertheim JO, Murrell B, Smith MD, Kosakovsky Pond SL, Scheffler K. RELAX: Detecting relaxed selection in a phylogenetic framework. Mol Biol Evol. 2015;32:820-32

60. Chaw RC, Arensburger P, Clarke TH, Ayoub NA, Hayashi CY. Candidate egg case silk genes for the spider Argiope argentata from differential gene expression analyses. Insect Mol Biol. 2016;25:757-68.

61. Fernández R, Kallal RJ, Dimitrov D, Ballesteros JA, Arnedo MA, Giribet G, et al. Phylogenomics, diversification dynamics, and comparative transcriptomics across the spider tree of life. Curr Biol. 2018;28(1489-1497):e5.

62. Aiken M, Coyle FA. Habitat disritbution, life history and behavior of Tetragnatha spider species in the Great Smoky Mountains National Park. J Arachnol. 2000;28:97-106.

63. Shinkai E, Takano S. A field guide to the spiders of Japan. Tokyo: Tokai University Press; 1984

64. Jose KS, Davis S, Sudhikumar AV, Sebastian PA. Redescription of Tetragnatha viridorufa Gravely from Kerala, India, Araneae: Tetragnathidae. J Bombay Nat Hist Soc. 2004;101:182-4.

65. Jeffery F, La Mattina C, Tuton-Blasingame T, Hsia Y, Gnesa E, Zhao L, et al. Microdissection of black widow spider silk-producing glands. J Vis Exp JoVE. 2011. https://doi.org/10.3791/2382.

66. Kono N, Nakamura H, Ito Y, Tomita M, Arakawa K. Evaluation of the impact of RNA preservation methods of spiders for de novo transcriptome assembly. Mol Ecol Resour. 2016;16:662-72.

67. Bolger AM, Lohse M, Usadel B. Trimmomatic: a flexible trimmer for Illumina sequence data. Bioinformatics. 2014;30:2114-20.

68. Grabherr MG, Haas BJ, Yassour M, Levin JZ, Thompson DA, Amit I, et al. Trinity: reconstructing a full-length transcriptome without a genome from RNA-Seq data. Nat Biotechnol. 2011;29:644-52.

69. Langmead B, Salzberg SL. Fast gapped-read alignment with Bowtie 2. Nat Methods. 2012;9:357-9.

70. Simão FA, Waterhouse RM, loannidis P, Kriventseva EV, Zdobnov EM. BUSCO: assessing genome assembly and annotation completeness with single-copy orthologs. Bioinformatics. 2015;31:3210-2.

71. Buchfink B, Xie C, Huson DH. Fast and sensitive protein alignment using DIAMOND. Nat Methods. 2015;12:59-60.

72. LiW, Godzik A. Cd-hit: a fast program for clustering and comparing large sets of protein or nucleotide sequences. Bioinformatics. 2006;22:1658-9.

73. Emms DM, Kelly S. OrthoFinder: solving fundamental biases in whole genome comparisons dramatically improves orthogroup inference accuracy. Genome Biol. 2015;16:157.

74. Kocot KM, Citarella MR, Moroz LL, Halanych KM. PhyloTreePruner: a phylogenetic tree-based approach for selection of orthologous sequences for phylogenomics. Evol Bioinforma. 2013;9:EBO.S12813.

75. Patro R, Duggal G, Love MI, Irizarry RA, Kingsford C. Salmon: fast and biasaware quantification of transcript expression using dual-phase inference. Nat Methods. 2017;14:417-9.

76. Soneson C, Love MI, Robinson MD. Differential analyses for RNA-seq: transcript-level estimates improve gene-level inferences. F1000Research. 2016. https://doi.org/10.12688/f1000research.7563.2.

77. Love MI, Huber W, Anders S. Moderated estimation of fold change and dispersion for RNA-seq data with DESeq2. Genome Biol. 2014;15:550.

78. Götz S, García-Gómez JM, Terol J, Williams TD, Nagaraj SH, Nueda MJ, et al. High-throughput functional annotation and data mining with the Blast2GO suite. Nucleic Acids Res. 2008;36:3420-35. 
79. Wright RM, Aglyamova GV, Meyer E, Matz MV. Gene expression associated with white syndromes in a reef building coral, Acropora hyacinthus. BMC Genomics. 2015;16:371.

80. Moretti S, Armougom F, Wallace IM, Higgins DG, Jongeneel CV, Notredame C. The M-Coffee web server: a meta-method for computing multiple sequence alignments by combining alternative alignment methods. Nucleic Acids Res. 2007;35(Suppl_2):W645-8.

81. Huelsenbeck JP, Ronquist F. MRBAYES: Bayesian inference of phylogenetic trees. Bioinformatics. 2001:17:754-5.

82. Letunic I, Bork P. Interactive tree of life (iTOL) v3: an online tool for the display and annotation of phylogenetic and other trees. Nucleic Acids Res. 2016;44:W242-5.

83. Zobel-Thropp PA, Bulger EA, Cordes MHJ, Binford GJ, Gillespie RG, Brewer MS. Sexually dimorphic venom proteins in long-jawed orb-weaving spiders (Tetragnatha) comprise novel gene families. PeerJ. 2018;6:e4691.

84. Miele V, Penel S, Duret L. Ultra-fast sequence clustering from similarity networks with SiLiX. BMC Bioinformatics. 2011;12:116.

85. Katoh $\mathrm{K}$, Asimenos $\mathrm{G}$, Toh $\mathrm{H}$. Multiple alignment of DNA sequences with MAFFT. In: Posada D, editor. Bioinformatics for DNA Sequence Analysis. Totowa, NJ: Humana Press; 2009. p. 39-64. doi:https://doi.org/10.1007/ 978-1-59745-251-9_3.

86. Price MN, Dehal PS, Arkin AP. FastTree: computing large minimum evolution trees with profiles instead of a distance matrix. Mol Biol Evol. 2009;26:1641-50.
87. Murrell B, Moola S, Mabona A, Weighill T, Sheward D, Kosakovsky Pond SL, et al. FUBAR: a fast, unconstrained bayesian approximation for inferring selection. Mol Biol Evol. 2013;30:1196-205.

88. Smith MD, Wertheim JO, Weaver S, Murrell B, Scheffler K, Kosakovsky Pond SL. Less is more: an adaptive branch-site random effects model for efficient detection of episodic diversifying selection. Mol Biol Evol. 2015;32:1342-53.

89. Mirarab S, Reaz R, Bayzid MS, Zimmermann T, Swenson MS, Warnow T. ASTRAL: genome-scale coalescent-based species tree estimation. Bioinformatics. 2014;30:1541-8.

90. Suyama M, Torrents D, Bork P. PAL2NAL: robust conversion of protein sequence alignments into the corresponding codon alignments. Nucleic Acids Res. 2006;34(Suppl_2):W609-12.

91. Yang Z. PAML 4: phylogenetic analysis by maximum likelihood. Mol Biol Evol. 2007;24:1586-91.

92. Nguyen L-T, Schmidt HA, von Haeseler A, Minh BQ. IQ-TREE: a fast and effective stochastic algorithm for estimating maximum-likelihood phylogenies. Mol Biol Evol. 2015;32:268-74.

\section{Publisher's Note}

Springer Nature remains neutral with regard to jurisdictional claims in published maps and institutional affiliations.
Ready to submit your research? Choose BMC and benefit from:

- fast, convenient online submission

- thorough peer review by experienced researchers in your field

- rapid publication on acceptance

- support for research data, including large and complex data types

- gold Open Access which fosters wider collaboration and increased citations

- maximum visibility for your research: over 100M website views per year

At BMC, research is always in progress.

Learn more biomedcentral.com/submissions 